\title{
SOLITON DYNAMICS FOR THE SCHRÖDINGER-NEWTON SYSTEM
}

\author{
PIETRO D'AVENIA AND MARCO SQUASSINA
}

\begin{abstract}
We investigate the soliton dynamics for the Schrödinger-Newton system by proving a suitable modulational stability estimates in the spirit of those obtained by Weinstein for local equations.
\end{abstract}

\section{INTRODUCTION}

Let us consider the Schrödinger-Newton system

$$
\left\{\begin{array}{l}
i \hbar \partial_{t} u=-\frac{\hbar^{2}}{2 m} \Delta u+V(x) u-\phi u \\
-\Delta \phi=4 \pi \gamma|u|^{2}
\end{array}\right.
$$

where $u:[0, \infty) \times \mathbb{R}^{3} \rightarrow \mathbb{C}, \phi: \mathbb{R}^{3} \rightarrow \mathbb{R}$ is the gravitational potential, $V: \mathbb{R}^{3} \rightarrow \mathbb{R}$ is an external potential, $m$ is the mass of the particle, $\gamma=G m^{2}$, where $G$ is the Newton constant. Up to suitable rescalings, (1.1) can be rewritten as the equation

$$
i \varepsilon \partial_{t} u^{\varepsilon}=-\frac{\varepsilon^{2}}{2} \Delta u^{\varepsilon}+V(x) u^{\varepsilon}-\frac{1}{\varepsilon^{2}}\left(\frac{1}{|x|} *\left|u^{\varepsilon}\right|^{2}\right) u^{\varepsilon} \quad \text { in }[0, \infty) \times \mathbb{R}^{3} .
$$

This equation was originally elaborated by Pekar [23] around 1954 in the framework of quantum mechanics. Subsequently, in 1976, Choquard [16] adopted the equation as an approximation of the Hartree-Fock theory. More recently, in 1996, Penrose [24] settled it as a model of self-gravitating matter. From the point of view of global well-posedness and smoothness for arbitrary initial data $u_{0}^{\varepsilon} \in H^{1}\left(\mathbb{R}^{3}, \mathbb{C}\right)$, the Cauchy problem associated with (1.2) was completely investigated in [6]. Concerning the existence and qualitative properties of the associated standing wave solutions, we refer the reader to the classical contributions by Lions $[18,19]$ on concentration compactness (see also [22] for a more general situation). For what regards orbital stability of solutions to $(1.2)$ - for a fixed $\varepsilon$ - and with respect to a suitable family of ground states, we refer to the contribution due to Cazenave and Lions [7, Theorem IV.2] and those by Grillakis and Shatah [12,13]. Years later, in the frame of stability theory for local Schrödinger equation

$$
i \varepsilon \partial_{t} u^{\varepsilon}=-\frac{\varepsilon^{2}}{2} \Delta u^{\varepsilon}+V(x) u^{\varepsilon}-\left|u^{\varepsilon}\right|^{2 p} u^{\varepsilon} \quad \text { in }[0, \infty) \times \mathbb{R}^{3}, \quad 0<p<\frac{2}{3},
$$

several contributions appeared about the study of the so called semi-classical (or point particle) limit behaviour as the parameter $\varepsilon$ vanishes, both for the standing waves and the full evolutionary problem. Concerning the former, for local equations we refer to the monograph by Ambrosetti and Malchiodi [1] and to the references therein, while for nonlocal equations, we refer to [8] and to the related references. About the latter, rigorous results about the soliton dynamics of local Schrödinger were obtained in various papers, among which we mention the contributions by Bronski and Jerrard [5] and Keraani [14] by means of arguments which are purely based on the use of conservation laws satisfied by the equation and by the associated Newtonian system $\ddot{x}(t)=-\nabla V(x(t))$, combined with the modulational stability estimates due to Weinstein $[28,29]$. With different techniques similar results were obtained in [10] by Fröhlich, Gustafson, Jonsson and Sigal (see also [9]). Roughly speaking, the soliton dynamics occurs when, choosing a suitable initial datum $u_{0}^{\varepsilon}(x)=r\left(\left(x-x_{0}\right) / \varepsilon\right)$ the corresponding solution $u^{\varepsilon}(t)$ mantains the shape $r((x-x(t)) / \varepsilon)$, up to an estimable error and locally in time, in the transition from quantum to classical mechanics, namely as

2000 Mathematics Subject Classification. 35Q51, 35Q40, 35Q41.

Key words and phrases. Soliton dynamics, Schrödinger-Newton system, modulational stability, ground states.

Both the authors were supported by 2009 MIUR project: "Variational and Topological Methods in the Study of Nonlinear Phenomena". Moreover, the first author was supported also by the 2012 INDAM project "Metodi Variazionali e Problemi Ellittici Non Lineari" and 2011 FRA project "Onde solitarie". This work has been partially carried out during a stay of P. d'Avenia in Verona. He would like to express his deep gratitude to the Dipartimento di Informatica for the warm hospitality. 
$\varepsilon \rightarrow 0$. For a nice survey on solitons and their stability features, see the work by Tao [25]. In the nonlocal case, the semiclassical limit of the standing waves of (1.2) was recently studied by Wei and Winter [27]. The full evolution problem (1.2) was studied in a soliton dynamics regime by Fröhlich, Tsai and Yau in [11] along the line followed in [10] for the local case. On the contrary, to the best of the authors' knowledge, there is no nonlocal counterpart of the study of point particle dynamics along the technique initiated in the work by Bronski and Jerrard [5]. This is precisely the aim of this paper. Let $r \in H^{1}\left(\mathbb{R}^{3}\right)$ be the unique radial, positive solution of

$$
-\frac{1}{2} \Delta r+r-\left(\frac{1}{|x|} *|r|^{2}\right) r=0
$$

The main tool exploited in $[5,14]$ in the local case $(1.3)$ is a kind of coercivity estimate for the differences $\mathcal{E}(\phi)-\mathcal{E}(r)$ upon suitable complex-valued functions $\phi$ such that $\|\phi\|_{2}=\|r\|_{2}$ for the energy functional $\mathcal{E}(\phi)=\|\nabla \phi\|_{2}^{2} / 2-\|\phi\|_{2 p+2}^{2 p+2} /(2 p+2)$ associated with $-\Delta \phi / 2+\phi=|\phi|^{2 p} \phi$ on $\mathbb{R}^{3}$, obtained by exploiting the spectral properties of its linearized operator. The first main result of the paper is the validity of this property for the nonlocal equation (1.4). Precisely, let $\mathcal{E}: H^{1}\left(\mathbb{R}^{3}, \mathbb{C}\right) \rightarrow \mathbb{R}$ be the energy functional defined by

$$
\mathcal{E}(\phi)=\frac{1}{2} \int|\nabla \phi|^{2}-\frac{1}{2} \iint \frac{|\phi(x)|^{2}|\phi(y)|^{2}}{|x-y|}
$$

and $\|\cdot\|$ denote the $H^{1}\left(\mathbb{R}^{3}, \mathbb{C}\right)$-norm. Then we have the following

Theorem 1.1. There exists a positive constant $C$ such that

$$
\mathcal{E}(\phi)-\mathcal{E}(r) \geq C \inf _{x \in \mathbb{R}^{3}, \theta \in[0,2 \pi)}\left\|\phi-e^{i \theta} r(\cdot-x)\right\|^{2}+o\left(\inf _{x \in \mathbb{R}^{3}, \theta \in[0,2 \pi)}\left\|\phi-e^{i \theta} r(\cdot-x)\right\|^{2}\right),
$$

for every $\phi \in H^{1}\left(\mathbb{R}^{3}, \mathbb{C}\right)$ such that $\|\phi\|_{2}=\|r\|_{2}$ and $\inf _{x \in \mathbb{R}^{3}, \theta \in[0,2 \pi)}\left\|\phi-e^{i \theta} r(\cdot-x)\right\| \leq\|r\|$.

By combining Lions's concentration-compactness [18, 19] with [7, (ii) of Theorem IV.1] and recalling the uniqueness of the ground state $r$, an equivalent formulation of Theorem 1.1 could be given by dropping the $o(\cdot)$ term and adding instead the requirement that the difference $\mathcal{E}(\phi)-\mathcal{E}(r)$ be small enough. For local Schrödinger equations with power nonlinearity, Theorem 1.1 was proved in [28,29] while [21] contains a proof for the result for one dimensional Schrödinger systems. We shall prove Theorem 1.1 in Section 2 by virtue of a careful study of the (real and imaginary) linearized operators $L_{-}$and $L_{+}$associated with (1.4) on some subspaces of $H^{1}\left(\mathbb{R}^{3}, \mathbb{C}\right.$ ) defined by suitable orthogonality conditions. Once the estimate of Theorem 1.1 holds true, a natural application is to obtain the soliton dynamics behaviour, in the semi-relativistic limit $\varepsilon \rightarrow 0$, for the Cauchy problem

$$
\left\{\begin{array}{l}
i \varepsilon \partial_{t} u^{\varepsilon}=-\frac{\varepsilon^{2}}{2} \Delta u^{\varepsilon}+V(x) u^{\varepsilon}-\frac{1}{\varepsilon^{2}}\left(\frac{1}{|x|} *\left|u^{\varepsilon}\right|^{2}\right) u^{\varepsilon} \\
u^{\varepsilon}(0, x)=r\left(\frac{x-x_{0}}{\varepsilon}\right) e^{\frac{i}{\varepsilon} x \cdot v_{0}}
\end{array}\right.
$$

where and $x_{0} \in \mathbb{R}^{3}$ and $v_{0} \in \mathbb{R}^{3}$ are, respectively, the initial position and velocity of

$$
\left\{\begin{array}{l}
\dot{x}(t)=v(t), \\
\dot{v}(t)=-\nabla V(x(t)), \\
x(0)=x_{0}, \\
v(0)=v_{0} .
\end{array}\right.
$$

Problem (1.5) is globally well-posed, provided that $V \in L^{m}\left(\mathbb{R}^{3}\right)+L^{\infty}\left(\mathbb{R}^{3}\right)$, for some $m>3 / 2[6$, Corollary 6.1.2 and Example 1]. Denoting $\|\cdot\|_{\mathcal{H}_{\varepsilon}}^{2}=\frac{1}{\varepsilon}\|\nabla \cdot\|_{2}^{2}+\frac{1}{\varepsilon^{3}}\|\cdot\|_{2}^{2}$, we prove the following

Theorem 1.2. Assume that $V=V_{1}+V_{2}$ with $V_{1} \in \mathcal{C}^{3}\left(\mathbb{R}^{3}\right)$ and $D^{2} V_{2} \in C^{2}\left(\mathbb{R}^{3}\right)$, where $V_{2}$ is bounded from below. Therefore, for every $\varepsilon$ small, we have

$$
\left\|u^{\varepsilon}(t, x)-r\left(\frac{x-x(t)}{\varepsilon}\right) e^{i \frac{v(t) \cdot x}{\varepsilon}}\right\|_{\mathcal{H}_{\varepsilon}}=\mathcal{O}(\varepsilon),
$$

on finite time intervals. 
We shall prove Theorem 1.2 in Section 3 by showing a few preliminary facts about the energy expansion and the momentum identity for (1.5) and then exploiting Theorem 1.1 on a suitable auxiliary function related to the solution of (1.5). Once that stage is achieved, the argument to get the uniform bound on the error - on finite time intervals - follows as in [5,14]. Quite recently, Benci, Ghimenti and Micheletti in [2,3] obtained, for a variant of the local equation (1.3), a soliton dynamics behaviour with error estimate on the whole $[0, \infty)$ and, in general, working for equations whose ground states need not be unique or nondegenerate. In a forthcoming paper, we aim to use their technique on a general nonlocal problem for which uniqueness and nondegeneracy results are not available yet.

\section{Notations.}

(1) If $u, v \in \mathbb{C}, u \cdot v=\operatorname{Re}(u \bar{v})=\frac{1}{2}(u \bar{v}+v \bar{u})$.

(2) $H^{1}\left(\mathbb{R}^{3}\right)=H^{1}\left(\mathbb{R}^{3}, \mathbb{R}\right)$ and $H^{1}\left(\mathbb{R}^{3}, \mathbb{C}\right)$ are the Sobolev spaces endowed with the norm $\|\cdot\|=\left(\|\cdot\|_{2}^{2}+\right.$ $\left.\frac{1}{2}\|\nabla \cdot\|_{2}^{2}\right)^{1 / 2}$.

(3) If $u, v \in H^{1}\left(\mathbb{R}^{3}, \mathbb{C}\right)$ we denote with $(u, v)$ the scalar product in $L^{2}\left(\mathbb{R}^{3}, \mathbb{C}\right)$ and with $(u, v)_{H^{1}}=$ $(u, v)+\frac{1}{2}(\nabla u, \nabla v)$.

(4) $\mathcal{C}^{3}\left(\mathbb{R}^{3}\right)$ is the space of functions $u \in C^{3}\left(\mathbb{R}^{3}\right)$ with $\left\|D^{\alpha} u\right\|_{\infty}<\infty$ for any $|\alpha| \leq 3$.

(5) $C$ denotes a generic positive constant which can changes from line to line.

\section{Proof of Theorem 1.1}

2.1. Preliminary tools. In this section we collect a few basic properties about the ground state solutions to (1.4) and its corresponding linearized operator.

2.1.1. The limit problem. Let us consider the eigenvalue problem

$$
-\frac{1}{2} \Delta \varphi-\left(\frac{1}{|x|} *|\varphi|^{2}\right) \varphi=e \varphi .
$$

A fundamental tool in our analysis is the following result due to Lieb [16, Theorem 8].

Theorem 2.1. If $\varphi \in H^{1}\left(\mathbb{R}^{3}\right),\|\varphi\|_{2}=\lambda$ and $\mathcal{E}(\varphi)=\inf \left\{\mathcal{E}(\phi) \mid \phi \in H^{1}\left(\mathbb{R}^{3}\right),\|\phi\|_{2}=\lambda\right\}$, then $\varphi$ satisfies equation (2.1) for some e $<0$. Moreover if $\varphi \in H^{1}\left(\mathbb{R}^{3}, \mathbb{C}\right.$ ) satisfies (2.1) (not necessarily a minimizer) for an arbitrary Lagrange multiplier e, then:

(i) $|x|^{-1} *|\varphi|^{2} \in L^{p}\left(\mathbb{R}^{3}\right)$, for every $4 \leq p \leq \infty$;

(ii) $\left(|x|^{-1} *|\varphi|^{2}\right) \varphi \in L^{p}\left(\mathbb{R}^{3}, \mathbb{C}\right)$, for every $\frac{4}{3} \leq p \leq 6$;

(iii) $|x|^{-1} *|\varphi|^{2}$ is a continuous function which goes to zero at infty;

(iv) If $e<0$ then $\varphi \in C^{\infty}\left(\mathbb{R}^{3}\right)$ and goes to zero at infinity (and hence $\varphi$ is a classical solution of (2.1)).

Moreover we also need the following

Proposition 2.2. Let $r$ be the unique positive and radial solution of (1.4). We have that:

(i) $r$ has a nondegenerate linearization (the linearization of (1.4) around $r$ has a nullspace that is entirely due to the equations invariance under phase and translation transformation);

(ii) $r(0)=\max _{x \in \mathbb{R}^{3}} r(x)$ and if we take $r(x)=r_{0}(|x|)$, we have that $r_{0}$ is strictly decreasing and

$$
\lim _{|x| \rightarrow \infty} r_{0}(|x|) e^{|x|}|x|=\lambda_{0}>0, \quad \lim _{|x| \rightarrow \infty} \frac{r_{0}^{\prime}(|x|)}{r_{0}(|x|)}=-1 ;
$$

(iii) $r$ can be obtained as the minimum point of $\mathcal{E}$ in $\mathcal{M}=\left\{u \in H^{1}\left(\mathbb{R}^{3}\right) \mid\|u\|_{2}=\|r\|_{2}\right\}$.

Proof. For the proof of $(i)$ and $(i i)$ we refer to $[15,16,20,26]$. Here, for the sake of completeness, we prove $($ iii $)$. We know that for every $\alpha>0, \mathcal{E}$ has a unique radial and strictly positive minimum point on $\left\{u \in H^{1}\left(\mathbb{R}^{3}\right) \mid\|u\|_{2}=\alpha\right\}$ (see [16, Theorem 7 and Theorem 10]). Let $\bar{u}$ be such minimum point on $\mathcal{M}$. There exists $\lambda>0$ such that

$$
-\frac{1}{2} \Delta \bar{u}-\left(\frac{1}{|x|} *|\bar{u}|^{2}\right) \bar{u}=-\lambda \bar{u} .
$$

It is easy to show that $\lambda^{-1} \bar{u}\left(\lambda^{-1 / 2} x\right)$ is a radial and strictly positive solution of (1.4). Then, by the uniqueness, we have that $r(x)=\lambda^{-1} \bar{u}\left(\lambda^{-1 / 2} x\right)$ and, since $\|\bar{u}\|_{2}^{2}=\|r\|_{2}^{2}=\|\bar{u}\|_{2}^{2} / \sqrt{\lambda}$, we get $\lambda=1$. 
2.1.2. The linearized problem. Let $r$ be the unique radial positive solution of (1.4) and consider the linearized operator $L$ for $(1.4)$ at $r$, acting on $L^{2}\left(\mathbb{R}^{3}, \mathbb{C}\right)$ with domain in $H^{2}\left(\mathbb{R}^{3}, \mathbb{C}\right)$,

$$
L \xi=-\frac{1}{2} \Delta \xi+\xi-\left(\frac{1}{|x|} * r^{2}\right) \xi-\left(\frac{1}{|x|} *(r(\xi+\bar{\xi}))\right) r .
$$

We can write

$$
L=\left(\begin{array}{cc}
L_{+} & 0 \\
0 & L_{-}
\end{array}\right)
$$

where $L_{+}$and $L_{-}$act respectively on the real and imaginary part of $\xi$, i.e. if $\eta$ is real

$$
L_{+} \eta=-\frac{1}{2} \Delta \eta+\eta-\left(\frac{1}{|x|} * r^{2}\right) \eta-2\left(\frac{1}{|x|} *(r \eta)\right) r \quad \text { and } \quad L_{-} \eta=-\frac{1}{2} \Delta \eta+\eta-\left(\frac{1}{|x|} * r^{2}\right) \eta .
$$

It can be proved (see [15]) that

$$
\begin{aligned}
& \operatorname{Ker} L_{+}=\operatorname{span}\left\{\partial_{x_{1}} r, \partial_{x_{2}} r, \partial_{x_{3}} r\right\}, \\
& \operatorname{Ker} L_{-}=\operatorname{span}\{r\} .
\end{aligned}
$$

2.2. Preliminary results. Let us set

$$
\Xi_{j}(r):=\partial_{x_{j}}\left(\left(|x|^{-1} * r^{2}\right) r\right)=\left(|x|^{-1} * r^{2}\right) \partial_{x_{j}} r+2\left(|x|^{-1} *\left(r \partial_{x_{j}} r\right)\right) r, \quad \text { for } j=1,2,3 .
$$

Notice that $\Xi_{j}(r) \in L^{2}\left(\mathbb{R}^{3}\right)$. Indeed, for the first term it is enough to observe that $|x|^{-1} * r^{2} \in L^{\infty}\left(\mathbb{R}^{3}\right)$, by (i) of Theorem 2.1. Moreover, writing $|x|^{-1}=h_{1}+h_{2}$ with $h_{1} \in L^{\infty}\left(\mathbb{R}^{3}\right)$ and $h_{2} \in L^{3 / 2}\left(\mathbb{R}^{3}\right)$ yields

$$
\left\|\left(|x|^{-1} *\left(r \partial_{x_{j}} r\right)\right) r\right\|_{2}^{2} \leq 2\left\|h_{1}\right\|_{\infty}^{2}\|r\|_{2}^{4}\left\|\partial_{x_{j}} r\right\|_{2}^{2}+2\left\|h_{2}\right\|_{3 / 2}^{2}\|r\|_{6}^{4}\left\|\partial_{x_{j}} r\right\|_{2}^{2}
$$

We shall prove the following

Proposition 2.3. Let $w \in H^{1}\left(\mathbb{R}^{3}, \mathbb{C}\right)$ and $u$ and $v$ be the real and the imaginary part of $w$. Let us assume that $\|w+r\|_{2}=\|r\|_{2}$ and

$$
\left(u, \Xi_{j}(r)\right)=0, \quad \text { for } j=1,2,3 .
$$

Then, there exist positive constants $D, D_{h}$ such that

$$
\left(L_{+} u, u\right) \geq D\|u\|^{2}-D_{1}\|w\|^{4}-D_{2}\|w\|^{3} .
$$

In order to prove Proposition 2.3 we proceed by proving some preliminary results. Let us set

$$
\mathcal{V}=\left\{u \in H^{1}\left(\mathbb{R}^{3}\right) \mid(u, r)=0\right\} .
$$

Lemma 2.4. $\inf _{\mathcal{V}}\left(L_{+} u, u\right)=0$.

Proof. Since $r$ is the minimum point of

$$
I(u)=\mathcal{E}(u)+\|u\|_{2}^{2}
$$

on $\mathcal{M}$ (defined in (iii) of Proposition 2.2), then, for every smooth curve $\varphi:[-1,1] \rightarrow \mathcal{M}$ such that $\varphi(0)=r$ we have that

$$
\left.\frac{d^{2} I(\varphi(s))}{d s^{2}}\right|_{s=0} \geq 0
$$

Therefore, being $I^{\prime}(r)=0$, we get

$$
0 \leq\left\langle I^{\prime \prime}(r) \varphi^{\prime}(0), \varphi^{\prime}(0)\right\rangle=2\left(L_{+} \varphi^{\prime}(0), \varphi^{\prime}(0)\right)
$$

Since the map $s \rightarrow\|\varphi(s)\|_{2}$ is constant, we have that $\varphi^{\prime}(0) \in \mathcal{V}$. Then, by the arbitrariness of $\varphi^{\prime}(0)$, we can say that $\inf _{\mathcal{V}}\left(L_{+} u, u\right) \geq 0$. On the other hand, for every $j=1,2,3$ we have that $\partial_{x_{j}} r \in \mathcal{V}$ and $\left(L_{+} \partial_{x_{j}} r, \partial_{x_{j}} r\right)=0$ and then we conclude.

Lemma 2.5. There exists $C>0$ such that

$$
\begin{aligned}
& \int\left(|x|^{-1} * r^{2}\right) u^{2} \leq C\|u\|_{2}^{2}, \quad \text { for all } u \in L^{2}\left(\mathbb{R}^{3}\right), \\
& \int\left(|x|^{-1} *(r u)\right) r u \leq C\|u\|_{2}^{2}, \quad \text { for all } u \in L^{2}\left(\mathbb{R}^{3}\right) .
\end{aligned}
$$


Proof. By $(i)$ of Theorem 2.1, inequality (2.6) easily follows. Moreover, combining the Hardy-LittlewoodSobolev [17, Theorem 4.3] and Hölder inequality, we get

$$
\int\left(|x|^{-1} *(r u)\right) r u \leq C\|r u\|_{6 / 5}\|r u\|_{6 / 5} \leq C\|r\|_{3}^{2}\|u\|_{2}^{2} \leq C\|u\|_{2}^{2},
$$

concluding the proof.

Moreover, we have the following

Lemma 2.6. Assume that $u_{n} \rightarrow u$ in $H^{1}\left(\mathbb{R}^{3}\right)$ as $n \rightarrow \infty$. Then, up to a subsequence, we have

$$
\begin{aligned}
\lim _{n} \int\left(|x|^{-1} * r^{2}\right) u_{n}^{2} & =\int\left(|x|^{-1} * r^{2}\right) u^{2}, \\
\lim _{n} \int\left(|x|^{-1} *\left(r u_{n}\right)\right) r u_{n} & =\int\left(|x|^{-1} *(r u)\right) r u .
\end{aligned}
$$

Proof. Up to a subsequence, $u_{n} \rightarrow u$ a.e. Since the sequence $\left\{u_{n}^{2}\right\}$ is bounded in $L^{6 / 5}\left(\mathbb{R}^{3}\right)$, up to a subsequence, it converges weakly to some $z \in L^{6 / 5}\left(\mathbb{R}^{3}\right)$. Taking into account the poinwise convergence of $\left\{u_{n}\right\}$ to $u$, it follows that $z=u^{2}$. Hence, in order to get (2.7), it is sufficient to have $|x|^{-1} * r^{2} \in L^{6}\left(\mathbb{R}^{3}\right)$ which follows from $(i)$ of Theorem 2.1 Concerning (2.8), we have

$$
\left|\int\left(|x|^{-1} *\left(r u_{n}\right)\right) r u_{n}-\int\left(|x|^{-1} *(r u)\right) r u\right| \leq I_{n}+J_{n},
$$

where we have set

$$
I_{n}=\left|\int\left(|x|^{-1} *\left(r u_{n}\right)\right)\left(r u_{n}-r u\right)\right|, \quad J_{n}=\left|\int\left(|x|^{-1} *\left(r u_{n}-r u\right)\right) r u\right| .
$$

Observe that, since $\left\{u_{n}^{6 / 5}\right\}$ converges weakly to $u^{6 / 5}$ in $L^{2}\left(\mathbb{R}^{3}\right)$ and $r^{6 / 5} \in L^{2}\left(\mathbb{R}^{3}\right)$, we have $\left\|r u_{n}\right\|_{6 / 5} \rightarrow$ $\|r u\|_{6 / 5}$. Since $r u_{n} \rightarrow r u$ in $L^{6 / 5}\left(\mathbb{R}^{3}\right)$ as $n \rightarrow \infty$, the uniform convexity of $L^{6 / 5}\left(\mathbb{R}^{3}\right)$ yields $\left\|r u_{n}-r u\right\|_{6 / 5} \rightarrow 0$ as $n \rightarrow \infty$. Therefore, from the Hardy-Littlewood-Sobolev inequality, we deduce

$$
I_{n} \leq C\left\|r u_{n}\right\|_{6 / 5}\left\|r u_{n}-r u\right\|_{6 / 5} \rightarrow 0 \quad \text { and } \quad J_{n} \leq C\left\|r u_{n}-r u\right\|_{6 / 5}\|r u\|_{6 / 5} \rightarrow 0,
$$

which concludes the proof.

Let us set

$$
\mathcal{V}_{0}=\left\{u \in H^{1}\left(\mathbb{R}^{3}\right) \mid(u, r)=\left(u, \Xi_{j}(r)\right)=0, j=1,2,3\right\} .
$$

Concerning the coercivity of $L_{+}$on $\mathcal{V}_{0}$, we have the following

Lemma 2.7. $\inf _{u \in \mathcal{V}_{0}} \frac{\left(L_{+} u, u\right)}{\|u\|^{2}}>0$.

Proof. We claim, first, that $\inf _{u \in \mathcal{V}_{0}} \frac{\left(L_{+} u, u\right)}{\|u\|_{2}^{2}}>0$. To this aim, let us consider

$$
\alpha:=\inf _{u \in \mathcal{V}_{0},\|u\|_{2}=1}\left(L_{+} u, u\right) .
$$

We want to prove that $\alpha>0$. Since $\mathcal{V}_{0} \subset \mathcal{V}$, then $\alpha \geq 0$ in light of Proposition 2.4. Suppose by contradiction that $\alpha=0$ and let $\left\{u_{n}\right\} \subset H^{1}\left(\mathbb{R}^{3}\right)$ be a minimizing sequence. By virtue of Lemma 2.5 , we readily have that $\left\{u_{n}\right\}$ is bounded in $H^{1}\left(\mathbb{R}^{3}\right)$. Then there exists $u \in H^{1}\left(\mathbb{R}^{3}\right)$ such that, up to a subsequence, $u_{n} \rightarrow u$ in $H^{1}\left(\mathbb{R}^{3}\right)$ and $u \in \mathcal{V}_{0}$. In turn, in light of Lemma 2.6, we deduce that

$$
\begin{aligned}
0 \leq\left(L_{+} u, u\right) & \leq \liminf _{n}\left(\left\|u_{n}\right\|^{2}-\int\left(|x|^{-1} * r^{2}\right) u_{n}^{2}-2 \int\left(|x|^{-1} *\left(r u_{n}\right)\right) r u_{n}\right) \\
& =\lim _{n}\left(L_{+} u_{n}, u_{n}\right)=0,
\end{aligned}
$$


so that $\left(L_{+} u, u\right)=0$. In turn, recalling that $\left(L_{+} u_{n}, u_{n}\right) \rightarrow 0$ as $n \rightarrow \infty$, we get

$$
\begin{aligned}
\|u\|^{2} & \leq \liminf _{n}\left\|u_{n}\right\|^{2} \leq \underset{n}{\limsup }\left\|u_{n}\right\|^{2} \\
& =\lim _{n}\left(\left(L_{+} u_{n}, u_{n}\right)+\int\left(|x|^{-1} * r^{2}\right) u_{n}^{2}+2 \int\left(|x|^{-1} *\left(r u_{n}\right)\right) r u_{n}\right) \\
& =\left(L_{+} u, u\right)+\int\left(|x|^{-1} * r^{2}\right) u^{2}+2 \int\left(|x|^{-1} *(r u)\right) r u=\|u\|^{2} .
\end{aligned}
$$

Then $\left\{u_{n}\right\}$ converges to $u$ in $H^{1}\left(\mathbb{R}^{3}\right)$ and $u$ solves the constrained minimization problem. Then there exist five Lagrange multipliers $\lambda, \mu, \gamma_{1}, \gamma_{2}, \gamma_{3} \in \mathbb{R}$ such that for every $\eta \in H^{1}\left(\mathbb{R}^{3}\right)$

$$
\left(L_{+} u, \eta\right)=\lambda(u, \eta)+\mu(r, \eta)+\sum_{j=1}^{3} \gamma_{j} \int \Xi_{j}(r) \eta .
$$

Since $\left(L_{+} u, u\right)=0$ and $u \in \mathcal{V}_{0}$, it follows immediately that $\lambda=0$. We claim that, for every $h=1,2,3$,

$$
0=\left(L_{+} u, \partial_{x_{h}} r\right)=\sum_{j=1}^{3} \gamma_{j} \int \Xi_{j}(r) \partial_{x_{h}} r=\gamma_{h} \int \Xi_{h}(r) \partial_{x_{h}} r .
$$

This follows by the following facts: $\left(r, \partial_{x_{h}} r\right)=0, L_{+}$is a self-adjoint operator, $\partial_{x_{h}} r \in \operatorname{Ker} L_{+}, r \in H^{2}\left(\mathbb{R}^{3}\right)$ and for every $j \neq h$ it holds

$$
\begin{aligned}
\int \Xi_{j}(r) \partial_{x_{h}} r & =\int \partial_{x_{j}}\left(\left(\frac{1}{|x|} * r^{2}\right) r\right) \partial_{x_{h}} r=\int \partial_{x_{j}}\left(-\frac{1}{2} \Delta r+r\right) \partial_{x_{h}} r \\
& =\frac{1}{2} \int \nabla \partial_{x_{j}} r \cdot \nabla \partial_{x_{h}} r+\int \partial_{x_{j}} r \partial_{x_{h}} r \\
& =\frac{1}{2} \int \frac{x_{j} x_{h}}{|x|^{4}}\left[\left(r_{0}^{\prime \prime}(|x|)|x|\right)^{2}-\left(r_{0}^{\prime}(|x|)\right)^{2}\right]+\int \frac{x_{j} x_{h}}{|x|^{2}}\left(r_{0}^{\prime}(|x|)\right)^{2}=0 .
\end{aligned}
$$

Moreover

$$
\int \Xi_{h}(r) \partial_{x_{h}} r=\int \partial_{x_{h}}\left(\left(\frac{1}{|x|} * r^{2}\right) r\right) \partial_{x_{h}} r=\int \partial_{x_{h}}\left(-\frac{1}{2} \Delta r+r\right) \partial_{x_{h}} r=\left\|\partial_{x_{h}} r\right\|^{2} .
$$

It follows that $\gamma_{h}=0$ for every $h=1,2,3$, yielding in turn

$$
\left(L_{+} u, \eta\right)=\mu(r, \eta), \quad \text { for every } \eta \in H^{1}\left(\mathbb{R}^{3}\right) .
$$

Now we claim that $\mu \neq 0$. Indeed if we suppose by contradiction that $\mu=0$, then, from (2.11), $u \in \operatorname{Ker} L_{+}$. Thus, from (2.2), we have that $u=\beta \cdot \nabla r$ with $\beta=\left(\beta_{1}, \beta_{2}, \beta_{3}\right) \in \mathbb{R}^{3}$. Moreover, since $u \in \mathcal{V}_{0}$, then, using (2.9) and (2.10), we have

$$
0=\int \Xi_{j}(r)(\beta \cdot \nabla r)=\beta_{j}\left\|\partial_{x_{j}} r\right\|^{2}, \quad \text { for every } j=1,2,3 .
$$

Then $\beta=0$, namely $u=0$, contradicting $\|u\|_{2}=1$. Notice now that

$$
L_{+}(x \cdot \nabla r)=-\Delta r+\sum_{j=1}^{3} x_{j} \partial_{x_{j}}\left[-\frac{1}{2} \Delta r+r-\left(\frac{1}{|x|} * r^{2}\right) r\right]=-\Delta r
$$

and, furthermore,

$$
L_{+}(r)=-2\left(|x|^{-1} * r^{2}\right) r
$$

Then

$$
L_{+}\left(-\frac{\mu}{2}(r+x \cdot \nabla r)\right)=\mu r=L_{+} u
$$

In turn, by the nondegeneracy of $r$ (see $(2.2)$ ), we learn that there exist $\vartheta=\left(\vartheta_{1}, \vartheta_{2}, \vartheta_{3}\right) \in \mathbb{R}^{3}$ with

$$
u=-\frac{\mu}{2}(r+x \cdot \nabla r)+\vartheta \cdot \nabla r
$$


We want to show that $\vartheta=0$. Since $u \in \mathcal{V}_{0}$, for every $j=1,2,3$, we have

$$
0=\int \Xi_{j}(r) u=-\frac{\mu}{2} \int \Xi_{j}(r) r-\frac{\mu}{2} \int \Xi_{j}(r) x \cdot \nabla r+\vartheta_{j}\left\|\partial_{x_{j}} r\right\|^{2}
$$

where we have used (2.9) and (2.10). On the other hand, we have

$$
\int \Xi_{j}(r) r=3 \int\left(\frac{1}{|x|} * r^{2}\right) r \partial_{x_{j}} r=3 \int\left(-\frac{1}{2} \Delta r+r\right) \partial_{x_{j}} r=\frac{3}{2} \int \nabla r \cdot \nabla\left(\partial_{x_{j}} r\right)+3 \int r \partial_{x_{j}} r=0
$$

and, since the map $x \mapsto\left(|x|^{-1} * r^{2}\right) r$ is radially symmetric,

$$
\int \Xi_{j}(r)(x \cdot \nabla r)=\int \partial_{x_{j}}\left[\left(\frac{1}{|x|} *|r|^{2}\right) r\right]|x| r_{0}^{\prime}(|x|)=0 .
$$

Then, for every $j=1,2,3$, we get $\vartheta_{j}\left\|\partial_{x_{j}} r\right\|^{2}=0$, yielding in turn $\vartheta=0$. Thus

$$
u=-\frac{\mu}{2}(r+x \cdot \nabla r) .
$$

But, since $u \in \mathcal{V}_{0}$,

$$
0=(u, r)=-\frac{\mu}{2}\left(\|r\|_{2}^{2}+(x \cdot \nabla r, r)\right) .
$$

Moreover, integrating by parts, we have

$$
(x \cdot \nabla r, r)=\frac{1}{2} \sum_{h=1}^{3} \int x_{h} \partial_{x_{h}}\left(r^{2}\right)=-\frac{3}{2}\|r\|_{2}^{2} .
$$

Dropping in (2.13) we get the contradiction and so that the proof of the claim is complete. Then, there exists a positive constant $\alpha_{0}>0$ such that

$$
\left(L_{+} u, u\right) \geq \alpha_{0}\|u\|_{2}^{2}, \quad \text { for every } u \in \mathcal{V}_{0} .
$$

If we put $\|u\| \|:=\sqrt{\left(L_{+} u, u\right)}$ for $u \in \mathcal{V}_{0}$, it is readily checked that \|\|$\cdot \|$ satisfies the required properties of a norm. Furthermore, if $\left\{u_{n}\right\}$ is a Cauchy sequence in $\left(\mathcal{V}_{0},|||\cdot|||\right)$, then, by $(2.14),\left\{u_{n}\right\}$ strongly converges to a function $u$ in $L^{2}\left(\mathbb{R}^{3}\right)$ and $u \in \mathcal{V}_{0}$. Moreover, using Lemma 2.5, we have that $\left\{u_{n}\right\}$ is a Cauchy sequence in $H^{1}\left(\mathbb{R}^{3}\right)$ and then $u$ has to be necessarily the strong limit in $H^{1}\left(\mathbb{R}^{3}\right)$. Therefore, $u_{n} \rightarrow u$ in $\left(\mathcal{V}_{0},|\|\cdot \mid\|)\right.$ and so we get that $\left(\mathcal{V}_{0}, \mid\|\cdot\| \|\right)$ is a Banach space and \|\|$\cdot \| \mid$ is equivalent to the norm of $H^{1}\left(\mathbb{R}^{3}\right)$. This concludes the proof.

Proof of Proposition 2.3. Since $\|w+r\|_{2}=\|r\|_{2}$, we have that

$$
\|r\|_{2}^{2}=\|r\|_{2}^{2}+\|w\|_{2}^{2}+2(u, r)
$$

and then

$$
(r, u)=-\frac{1}{2}\|w\|_{2}^{2}=-\frac{1}{2}\left(\|u\|_{2}^{2}+\|v\|_{2}^{2}\right)
$$

Without loss of generality, we can suppose that $\|r\|_{2}=1$. Let us write $u=u_{\|}+u_{\perp}$ where $u_{\|}=(u, r) r$. We notice that $u_{\perp}$ is orthogonal to $r$ in $L^{2}\left(\mathbb{R}^{3}\right)$ and, combining $(2.4)$ with $(2.12)$ we have that $\left(u_{\perp}, \Xi_{j}(r)\right)=0$ and namely $u_{\perp} \in \mathcal{V}_{0}$. Since $L_{+}$is selfadjoint, we have that

$$
\left(L_{+} u, u\right)=\left(L_{+} u_{\|}, u_{\|}\right)+2\left(L_{+} u_{\perp}, u_{\|}\right)+\left(L_{+} u_{\perp}, u_{\perp}\right) .
$$

So we study separately each term in the right hand side. By (2.15), the selfadjointness of $L_{+}$and since $r$ is solution of (1.4), we have that

$$
\left(L_{+} u_{\|}, u_{\|}\right)=\frac{1}{4}\|w\|_{2}^{4}\left(L_{+} r, r\right)=-\frac{1}{2}\|r\|^{2}\|w\|_{2}^{4}
$$

and

$$
\begin{aligned}
\left(L_{+} u_{\perp}, u_{\|}\right) & =-\frac{1}{2}\|w\|_{2}^{2}\left(u_{\perp}, L_{+} r\right)=\frac{1}{2}\|w\|_{2}^{2} \int \nabla u_{\perp} \cdot \nabla r=\frac{1}{2}\|w\|_{2}^{2}\left(\int \nabla u \cdot \nabla r-\int \nabla u_{\|} \cdot \nabla r\right) \\
& \geq-\frac{1}{2}\|w\|_{2}^{2}\|\nabla w\|_{2}\|\nabla r\|_{2} .
\end{aligned}
$$


Finally we notice that

$$
\|\nabla u\|_{2}^{2} \leq 2\left(\left\|\nabla u_{\|}\right\|_{2}^{2}+\left\|\nabla u_{\perp}\right\|_{2}^{2}\right)=\frac{1}{2}\|w\|_{2}^{4}\|\nabla r\|_{2}^{2}+2\left\|\nabla u_{\perp}\right\|_{2}^{2}
$$

so that

$$
\left\|\nabla u_{\perp}\right\|_{2}^{2} \geq \frac{1}{2}\|\nabla u\|_{2}^{2}-\frac{1}{4}\|w\|_{2}^{4}\|\nabla r\|_{2}^{2}
$$

Then, since $u_{\perp} \in \mathcal{V}_{0}$, applying Lemma 2.7 we have that

$$
\begin{aligned}
\left(L_{+} u_{\perp}, u_{\perp}\right) & \geq C\left\|u_{\perp}\right\|^{2}=C\left(\|u\|_{2}^{2}-\left\|u_{\|}\right\|_{2}^{2}+\left\|\nabla u_{\perp}\right\|_{2}^{2}\right) \\
& \geq C\left(\|u\|_{2}^{2}-|(u, r)|^{2}+\frac{1}{2}\|\nabla u\|_{2}^{2}-\frac{1}{4}\|w\|_{2}^{4}\|\nabla r\|_{2}^{2}\right) \\
& \geq C\left(\|u\|^{2}-\|w\|_{2}^{4}\right) .
\end{aligned}
$$

Combining (2.16), (2.17) and (2.18) we get (2.5).

Concerning the coercivity of $L_{-}$, we have the following

Proposition 2.8. $\inf _{v \neq 0,(v, r)_{H^{1}}=0} \frac{\left(L_{-} v, v\right)}{\|v\|^{2}}>0$.

Proof. Up to arguing as in the end of the proof of Lemma 2.7, it is enough to prove that

$$
\inf _{v \neq 0,(v, r)_{H^{1}}=0} \frac{\left(L_{-} v, v\right)}{\|v\|_{2}^{2}}>0
$$

Let us first prove that $L_{-}$is nonnegative. Since $|x|^{-1} \in L^{3-\delta}\left(\mathbb{R}^{3}\right)+L^{3+\delta}\left(\mathbb{R}^{3}\right)$ and $r^{2} \in L^{(3-\delta)^{\prime}}\left(\mathbb{R}^{3}\right) \cap$ $L^{(3+\delta)^{\prime}}\left(\mathbb{R}^{3}\right)$ for $\delta>0$ small, by applying [17, Lemma 2.20] we have that $x \mapsto|x|^{-1} * r^{2}$ goes to zero for $|x| \rightarrow \infty$ and so

$$
\lim _{|x| \rightarrow \infty}\left[1-\left(|x|^{-1} * r^{2}\right)\right]=1 .
$$

In turn, from [4, Theorem 3.1, p.165], we learn that $L_{-}$is bounded from below and has a discrete spectrum over $(-\infty, 1)$ which consists of eigenvalues of finite multiplicity. Moreover $r \in \operatorname{Ker} L_{-}$and so 0 is an eigenvalue of $L_{-}$and $r$ is a corresponding eigenfunction. But, from [4, Theorem 3.4, p.179], since the smallest eigenvalue of $L_{-}$is lower than 1 , then it is simple and the corresponding eigenfunction can be chosen to be positive everywhere. The positivity of $r$ implies that 0 is the smallest eigenvalue of $L_{-}$and $r$ is the corresponding eigenfunction. Thus, for any $v \in H^{1}\left(\mathbb{R}^{3}\right)$, we have that $\left(L_{-} v, v\right) \geq 0$. Let us consider

$$
\omega:=\inf _{(v, r)_{H^{1}}=0,\|v\|_{2}=1}\left(L_{-} v, v\right),
$$

and assume by contradiction that $\omega=0$. Let $\left\{v_{n}\right\} \subset H^{1}\left(\mathbb{R}^{3}\right)$ be a minimizing sequence. By virtue of (2.6), it follows that $\left\{v_{n}\right\}$ is bounded in $H^{1}\left(\mathbb{R}^{3}\right)$. Then there exists $v \in H^{1}\left(\mathbb{R}^{3}\right)$ such that, up to a subsequence, $v_{n} \rightarrow v$ in $H^{1}\left(\mathbb{R}^{3}\right)$ and $(v, r)_{H^{1}}=0$. In turn, in light of $(2.7)$, we deduce that

$$
0 \leq\left(L_{-} v, v\right) \leq \liminf _{n}\left(\left\|v_{n}\right\|^{2}-\int\left(|x|^{-1} * r^{2}\right) v_{n}^{2}\right)=\lim _{n}\left(L_{-} v_{n}, v_{n}\right)=0,
$$

so that $\left(L_{-} v, v\right)=0$. Hence, we obtain

$$
\begin{aligned}
\|v\|^{2} & \leq \liminf _{n}\left\|v_{n}\right\|^{2} \leq \limsup _{n}\left\|v_{n}\right\|^{2}=\lim _{n}\left(\left(L_{-} v_{n}, v_{n}\right)+\int\left(|x|^{-1} * r^{2}\right) v_{n}^{2}\right) \\
& =\left(L_{-} v, v\right)+\int\left(|x|^{-1} * r^{2}\right) v^{2}=\|v\|^{2} .
\end{aligned}
$$

Then $\left\{v_{n}\right\}$ converges to $v$ in $H^{1}\left(\mathbb{R}^{3}\right)$ which implies that $\|v\|_{2}=1$ and $v$ solves the minimization problem. In turn, there exist two Lagrange multipliers $\lambda, \mu \in \mathbb{R}$ such that, for every $\eta \in H^{1}\left(\mathbb{R}^{3}\right)$, it holds

$$
\left(L_{-} v, \eta\right)=\lambda(v, \eta)+\mu(r, \eta)_{H^{1}}
$$

Then, dropping $\eta=v$ into (2.19) immediately yields $\lambda=0$, so that, for any $\eta \in H^{1}\left(\mathbb{R}^{3}\right)$,

$$
\left(L_{-} v, \eta\right)=\mu(r, \eta)_{H^{1}} .
$$


Finally, by choosing now $\eta=r$ into equation $(2.20)$, and recalling that $L_{-} r=0$ yields

$$
0=\left(v, L_{-} r\right)=\left(L_{-} v, r\right)=\mu\|r\|^{2}
$$

where we used the fact that $L_{-}$is self-adjoint. Then $\mu=0$, namely $L_{-} v=0$. In light of $(2.3)$, there is $\vartheta \in \mathbb{R} \backslash\{0\}$ with $v=\vartheta r$. Thus $0=\vartheta\|r\|^{2}$ that is a contradiction. Then $\omega>0$ and the proof is complete.

For the proof of Theorem 1.1 we shall also need the following

Lemma 2.9. Let $\phi \in H^{1}\left(\mathbb{R}^{3}, \mathbb{C}\right)$ with $\|\phi\|_{2}=\|r\|_{2}$ and $\inf _{x \in \mathbb{R}^{3}, \theta \in[0,2 \pi)}\left\|\phi-e^{i \theta} r(\cdot-x)\right\| \leq\|r\|$. Then $\inf _{x \in \mathbb{R}^{3}, \theta \in[0,2 \pi)}\left\|\phi-e^{i \theta} r(\cdot-x)\right\|^{2}$ is achieved at some $x_{0} \in \mathbb{R}^{3}$ and $\gamma \in[0,2 \pi)$.

Proof. Consider the function $\Upsilon: \mathbb{R}^{3} \times[0,2 \pi) \rightarrow \mathbb{R}$ defined by setting

$$
\Upsilon(x, \theta)=\left\|\phi-e^{i \theta} r(\cdot-x)\right\|^{2}
$$

It is readily checked that $\Upsilon$ is continuous. Moreover, since $\|\phi\|_{2}=\|r\|_{2}$, we get

$$
\Upsilon(x, \theta)=2\|r\|_{2}^{2}+\frac{1}{2}\|\nabla r\|_{2}^{2}-2 \mathfrak{R e} \int e^{i \theta} \bar{\phi}(y) r(y-x) d y-\mathfrak{R e} \int e^{i \theta} \nabla \bar{\phi}(y) \cdot \nabla r(y-x) d y+\frac{1}{2}\|\nabla \phi\|_{2}^{2} .
$$

Taking into account that the families of functions $(r(\cdot-x))_{x \in \mathbb{R}^{3}}$ and $(\nabla r(\cdot-x))_{x \in \mathbb{R}^{3}}$ are bounded in $L^{2}\left(\mathbb{R}^{3}\right)$ and converge pointwise (almost everywhere) to zero as $|x| \rightarrow \infty$, it follows that they converge weakly to zero in $L^{2}\left(\mathbb{R}^{3}\right)$ as $|x| \rightarrow \infty$. In turn, it readily follows that, for any $\theta \in[0,2 \pi)$,

$$
\lim _{|x| \rightarrow \infty} \Upsilon(x, \theta)=2\|r\|_{2}^{2}+\frac{1}{2}\|\nabla r\|_{2}^{2}+\frac{1}{2}\|\nabla \phi\|_{2}^{2}>\|r\|^{2} .
$$

On the other hand, in light of the second assumption on the function $\phi$, for every $\delta>0$, there exist points $\tilde{x} \in \mathbb{R}^{3}$ and $\tilde{\theta} \in[0,2 \pi)$ such that $\Upsilon(\tilde{x}, \tilde{\theta}) \leq\|r\|^{2}+\delta$. It follows that the infimum of $\Upsilon$ over the unbounded set $\mathbb{R}^{3} \times[0,2 \pi)$ coincides with the infimum of $\Upsilon$ over the compact set $\bar{B}_{R}(0) \times[0,2 \pi]$ for every $R>0$ sufficiently large, yielding in turn the desired conclusion.

Proof of Theorem 1.1 concluded. Let $\phi \in H^{1}\left(\mathbb{R}^{3}, \mathbb{C}\right)$ be a function such that $\|\phi\|_{2}=\|r\|_{2}$ and inf $x \in \mathbb{R}^{3}, \theta \in[0,2 \pi) \| \phi-$ $e^{i \theta} r(\cdot-x)\|\leq\| r \|$. In light of Lemma 2.9 there exist $x_{0} \in \mathbb{R}^{3}, \gamma \in[0,2 \pi)$ with

$$
\inf _{x \in \mathbb{R}^{3}, \theta \in[0,2 \pi)}\left\|\phi-e^{i \theta} r(\cdot-x)\right\|^{2}=\left\|\phi-e^{i \gamma} r\left(\cdot-x_{0}\right)\right\|^{2} .
$$

Let us set $w(x):=e^{-i \gamma} \phi\left(x+x_{0}\right)-r(x)$. Denoting by $u$ and $v$ respectively the real and the imaginary part of $w$, we claim that $u$ satisfies $\left(u, \Xi_{j}(r)\right)=0$ for $j=1,2,3$ and $(v, r)_{H^{1}}=0$. Indeed, if, as in the proof of Lemma 2.9 , for any $\phi \in H^{1}\left(\mathbb{R}^{3}, \mathbb{C}\right), x \in \mathbb{R}^{3}, \theta \in \mathbb{R}$ we consider

$$
\begin{aligned}
\Upsilon(x, \theta) & =\left\|\phi-e^{i \theta} r(\cdot-x)\right\|^{2} \\
& =\|\phi\|^{2}+\|r\|^{2}-2 \mathfrak{R e} \int e^{i \theta} \bar{\phi}(y)\left(-\frac{1}{2} \Delta r+r\right)(y-x) d y \\
& =\|\phi\|^{2}+\|r\|^{2}-2 \mathfrak{R e} \int e^{i \theta} \bar{\phi}(y)\left[\left(|\cdot|^{-1} * r^{2}\right) r\right](y-x) d y,
\end{aligned}
$$

we have

$$
\frac{\partial \Upsilon}{\partial x_{j}}(x, \theta)=2 \mathfrak{R e} \int e^{i \theta} \bar{\phi}(y+x) \Xi_{j}(r)(y) d y \quad \text { and } \quad \frac{\partial \Upsilon}{\partial \theta}(x, \theta)=2 \mathfrak{I m} \int e^{i \theta} \bar{\phi}(y+x)\left(-\frac{1}{2} \Delta r+r\right)(y) d y .
$$

If $x=x_{0}$ and $\theta=\gamma$, since $e^{i \gamma} \bar{\phi}\left(\cdot+x_{0}\right)=\bar{w}+r, \partial_{x_{j}} \Upsilon\left(x_{0}, \gamma\right)=0$ and $\partial_{\theta} \Upsilon\left(x_{0}, \gamma\right)=0$, using (2.12), we get the orthogonality conditions. Then we consider the action $I(\phi)=\mathcal{E}(\phi)+\|\phi\|_{2}^{2}$ and we control the norm of $w$ in terms of the difference $I(\phi)-I(r)$. Using the scale invariance of $I$, recalling that $\left\langle I^{\prime}(r), w\right\rangle=0$ and using also

$$
\begin{aligned}
\left\langle I^{\prime \prime}(\zeta) \varsigma, \varsigma\right\rangle= & 2\|\varsigma\|^{2}-2 \int\left(|x|^{-1} *|\zeta|^{2}\right)|\varsigma|^{2} \\
& -4\left[\int\left(|x|^{-1} * \mathfrak{R e} \zeta \mathfrak{R e} \varsigma\right) \mathfrak{R e} \zeta \mathfrak{R e} \varsigma+2 \int\left(|x|^{-1} * \mathfrak{R e} \zeta \mathfrak{I m} \varsigma\right) \mathfrak{I m} \zeta \mathfrak{R e} \varsigma+\int\left(|x|^{-1} * \mathfrak{I m} \zeta \mathfrak{I m} \varsigma\right) \mathfrak{I m} \zeta \mathfrak{I m} \varsigma\right]
\end{aligned}
$$


for $\zeta, \varsigma \in H^{1}\left(\mathbb{R}^{3}, \mathbb{C}\right)$, the orthogonality conditions proved before, Propositions 2.3 and 2.8 , and the HardyLittlewood-Sobolev inequality we have

$$
\begin{aligned}
I(\phi)-I(r)= & I(r+w)-I(r)=\left\langle I^{\prime}(r), w\right\rangle+\frac{1}{2}\left\langle I^{\prime \prime}(r+\vartheta w) w, w\right\rangle \\
= & \|w\|^{2}-\int\left(|x|^{-1} *|r+\vartheta w|^{2}\right)|w|^{2}-2 \int\left(|x|^{-1} *(r+\vartheta u) u\right)(r+\vartheta u) u \\
& -2 \vartheta^{2} \int\left(|x|^{-1} * v^{2}\right) v^{2}-4 \vartheta \int\left(|x|^{-1} *(r+\vartheta u) v\right) u v \\
= & \left(L_{+} u, u\right)+\left(L_{-} v, v\right)-2 \vartheta \int\left(|x|^{-1} * r u\right)|w|^{2}-\vartheta^{2} \int\left(|x|^{-1} *|w|^{2}\right)|w|^{2} \\
& -4 \vartheta \int\left(|x|^{-1} * r u\right) u^{2}-2 \vartheta^{2} \int\left(|x|^{-1} * u^{2}\right) u^{2}-2 \vartheta^{2} \int\left(|x|^{-1} * v^{2}\right) v^{2} \\
& -4 \vartheta \int\left(|x|^{-1} * r v\right) u v-4 \vartheta^{2} \int\left(|x|^{-1} * u v\right) u v \\
\geq & D\|w\|^{2}-D_{1}\|w\|^{4}-D_{2}\|w\|^{3},
\end{aligned}
$$

which concludes the proof.

\section{Proof of Theorem 1.2}

3.1. Preliminary results. Let $u^{\varepsilon}$ be a solution of the Cauchy problem (1.5). The energy is defined as

$$
E_{\varepsilon}(t)=\frac{1}{2 \varepsilon} \int\left|\nabla u^{\varepsilon}(t, x)\right|^{2}+\frac{1}{\varepsilon^{3}} \int V(x)\left|u^{\varepsilon}(t, x)\right|^{2}-\frac{1}{2 \varepsilon^{5}} \iint \frac{\left|u^{\varepsilon}(t, x)\right|^{2}\left|u^{\varepsilon}(t, y)\right|^{2}}{|x-y|}
$$

and $E_{\varepsilon}(t)=E_{\varepsilon}(0)$ for every $t \geq 0$. Moreover the mass conservation reads as

$$
\frac{1}{\varepsilon^{3}} \int\left|u^{\varepsilon}(t, x)\right|^{2}=\|r\|_{2}^{2}=: m, \quad t \geq 0, \varepsilon>0 .
$$

For both conservations we refer the reader to [6, Theorem 4.3.1]. Setting

$$
\mathcal{H}(t):=\frac{1}{2} m|v(t)|^{2}+m V(x(t)), \quad t \geq 0,
$$

from system (1.6) it follows that $\mathcal{H}(t)=\mathcal{H}(0)$, for all $t>0$. We have the following

Lemma 3.1. $E_{\varepsilon}(t)=\mathcal{E}(r)+\mathcal{H}(t)+\mathcal{O}\left(\varepsilon^{2}\right)$ for all $t \in[0, \infty)$ and $\varepsilon>0$.

Proof. First, we observe that

$$
\left|\nabla\left(r\left(\frac{x-x_{0}}{\varepsilon}\right) e^{\frac{i}{\varepsilon} x \cdot v_{0}}\right)\right|^{2}=\frac{1}{\varepsilon^{2}}\left|\nabla r\left(\frac{x-x_{0}}{\varepsilon}\right)\right|^{2}+\frac{\left|v_{0}\right|^{2}}{\varepsilon^{2}} r^{2}\left(\frac{x-x_{0}}{\varepsilon}\right) .
$$

Then, by the conservation of energy $E_{\varepsilon}$, for any $t \in[0, \infty)$ and $\varepsilon>0$, there holds

$$
\begin{aligned}
E_{\varepsilon}(t)=E_{\varepsilon}(0) & =\frac{1}{2 \varepsilon^{3}} \int\left|\nabla r\left(\frac{x-x_{0}}{\varepsilon}\right)\right|^{2}+\frac{\left|v_{0}\right|^{2}}{2 \varepsilon^{3}} \int r^{2}\left(\frac{x-x_{0}}{\varepsilon}\right) \\
& +\frac{1}{\varepsilon^{3}} \int V(x) r^{2}\left(\frac{x-x_{0}}{\varepsilon}\right)-\frac{1}{2 \varepsilon^{5}} \iint \frac{r^{2}\left(\frac{x-x_{0}}{\varepsilon}\right) r^{2}\left(\frac{y-x_{0}}{\varepsilon}\right)}{|x-y|} \\
& =\frac{1}{2} \int|\nabla r|^{2}+\frac{1}{2} m\left|v_{0}\right|^{2}+\int V\left(x_{0}+\varepsilon x\right) r^{2}(x)-\frac{1}{2} \iint \frac{r^{2}(x) r^{2}(y)}{|x-y|} \\
& =\mathcal{E}(r)+\mathcal{H}(t)+\int V\left(x_{0}+\varepsilon x\right) r^{2}(x)-m V\left(x_{0}\right) .
\end{aligned}
$$

Taking into account that, since $\nabla^{2} V$ is bounded and

$$
\int x \nabla V\left(x_{0}\right) r^{2}(x)=0
$$

we have

$$
\int V\left(x_{0}+\varepsilon x\right) r^{2}(x)-m V\left(x_{0}\right)=\mathcal{O}\left(\varepsilon^{2}\right)
$$


and the assertion immediately follows.

Moreover we have

Lemma 3.2. There exists $C>0$ such that $\left\|\nabla u^{\varepsilon}(t)\right\|_{2} \leq C \sqrt{\varepsilon}$ for all $t \in[0, \infty)$ and $\varepsilon>0$.

Proof. Taking into account that $V$ is bounded from below, that $E_{\varepsilon}(0)$ is bounded with respect to $\varepsilon$ by Lemma 3.1 and the energy and mass are conserved quantities, there exists a positive constant $C$ independent of $\varepsilon$ such that, for all $t \in[0, \infty)$ and $\varepsilon>0$,

$$
\left\|\nabla u^{\varepsilon}(t)\right\|_{2}^{2} \leq \varepsilon C+\frac{1}{\varepsilon^{4}} \iint \frac{\left|u^{\varepsilon}(t, x)\right|^{2}\left|u^{\varepsilon}(t, x)\right|^{2}}{|x-y|} .
$$

Now, by the Hardy-Littlewood and Gagliardo-Nirenberg inequalities yields

$$
\frac{1}{\varepsilon^{4}} \iint \frac{\left|u^{\varepsilon}(x, t)\right|^{2}\left|u^{\varepsilon}(y, t)\right|^{2}}{|x-y|} \leq \frac{C}{\varepsilon^{4}}\left\|u^{\varepsilon}(t)\right\|_{12 / 5}^{4} \leq C \sqrt{\varepsilon}\left[\frac{\left\|u^{\varepsilon}(t)\right\|_{2}^{2}}{\varepsilon^{3}}\right]^{\frac{3}{2}}\left\|\nabla\left|u^{\varepsilon}(t)\right|\right\|_{2} \leq C \sqrt{\varepsilon}\left\|\nabla u^{\varepsilon}(t)\right\|_{2} .
$$

By combining the above inequalities the assertion follows.

First of all, let us define the momentum

$$
p^{\varepsilon}(t, x):=\frac{1}{\varepsilon^{2}} \mathfrak{I m}\left(\bar{u}^{\varepsilon}(t, x) \nabla u^{\varepsilon}(t, x)\right), \quad x \in \mathbb{R}^{3}, \quad t \in[0, \infty) .
$$

Lemma 3.3. The following identities hold

$$
\begin{array}{lll}
\partial_{t} \frac{\left|u^{\varepsilon}(t, x)\right|^{2}}{\varepsilon^{3}}=-\operatorname{div}\left(p^{\varepsilon}(t, x)\right), & t \in[0, \infty), x \in \mathbb{R}^{3}, \\
\partial_{t} \int p^{\varepsilon}(t, x)=-\frac{1}{\varepsilon^{3}} \int \nabla V(x)\left|u^{\varepsilon}(t, x)\right|^{2}, & t \in[0, \infty) .
\end{array}
$$

Proof. By multiplying the equation for $u^{\varepsilon}$ by $\bar{u}^{\varepsilon}$ and taking then the real part easily yields the first identity, via trivial manipulations. Concerning the second identity, since $u^{\varepsilon} \in C\left([0, \infty), H^{2}\left(\mathbb{R}^{3}\right)\right) \cap C^{1}\left([0, \infty), L^{2}\left(\mathbb{R}^{3}\right)\right)$ by [6, Theorem 5.2.1 and Remark 5.2.9] and the map $t \mapsto \int p_{j}^{\varepsilon}(t, x)(j=1,2,3)$ is $C^{1}([0, \infty))($ see e.g. [10, Appendix A]), we have

$$
\begin{aligned}
\partial_{t} \int p_{j}^{\varepsilon}(t, x)= & \frac{1}{\varepsilon^{2}} \int \mathfrak{I m}\left(\partial_{t} \bar{u}^{\varepsilon} \partial_{j} u^{\varepsilon}\right)-\frac{1}{\varepsilon^{2}} \int \mathfrak{I m}\left(\partial_{j} \bar{u}^{\varepsilon} \partial_{t} u^{\varepsilon}\right) \\
= & \frac{2}{\varepsilon^{2}} \int \mathfrak{I m}\left(\partial_{t} \bar{u}^{\varepsilon} \partial_{j} u^{\varepsilon}\right) \\
= & -\frac{1}{\varepsilon} \int \operatorname{div}\left(\partial_{j} \mathfrak{R e}\left(u^{\varepsilon}\right) \nabla \mathfrak{R e}\left(u^{\varepsilon}\right)\right)-\frac{1}{\varepsilon} \int \operatorname{div}\left(\partial_{j} \mathfrak{I m}\left(u^{\varepsilon}\right) \nabla \mathfrak{I m}\left(u^{\varepsilon}\right)\right)+\frac{1}{2 \varepsilon} \int \partial_{j}\left(\left|\nabla u^{\varepsilon}\right|^{2}\right) \\
& +\frac{1}{\varepsilon^{3}} \int V(x) \partial_{j}\left|u^{\varepsilon}\right|^{2}-\frac{1}{\varepsilon^{5}} \int\left(\frac{1}{|x|} *\left|\bar{u}^{\varepsilon}\right|^{2}\right) \partial_{j}\left|u^{\varepsilon}\right|^{2} .
\end{aligned}
$$

The first three terms as well as the last one in the above identity integrate to zero. Furthermore, the integral involving the nonlocal term is zero too, since it holds

$$
\begin{aligned}
\int\left(\frac{1}{|x|} *\left|\bar{u}^{\varepsilon}\right|^{2}\right) \partial_{j}\left|u^{\varepsilon}\right|^{2} & =-\int \partial_{j}\left(\frac{1}{|x|} *\left|\bar{u}^{\varepsilon}\right|^{2}\right)\left|u^{\varepsilon}\right|^{2}=-\int\left(\frac{1}{|x|} * \partial_{j}\left|\bar{u}^{\varepsilon}\right|^{2}\right)\left|u^{\varepsilon}\right|^{2} \\
& =-\int\left(\frac{1}{|x|} *\left|\bar{u}^{\varepsilon}\right|^{2}\right) \partial_{j}\left|u^{\varepsilon}\right|^{2}
\end{aligned}
$$

Hence, the assertion follows. 
3.2. Proof of Theorem 1.2 concluded. Once Theorem 1.1 holds true, the proof of Theorem 1.2 proceeds as in $[5,14]$. Given $T_{0}>0$ to be choosen suitably small, the function

$$
\Psi^{\varepsilon}(t, x):=e^{-\frac{i}{\varepsilon}(\varepsilon x+x(t)) \cdot v(t)} u^{\varepsilon}(t, \varepsilon x+x(t))
$$

satisfies $\left\|\Psi^{\varepsilon}(t, \cdot)\right\|_{2}=\|r\|_{2}$. Furthermore, taking into account Lemma 3.1, the conservation of $\mathcal{H}$ and the characterization of $r$ as infimum on $\mathcal{M}$, by a direct computation we end up with

$$
\begin{aligned}
0 \leq \mathcal{E}\left(\Psi^{\varepsilon}(t)\right)-\mathcal{E}(r) & =E_{\varepsilon}(t)+\frac{1}{2} m|v(t)|^{2}-v(t) \int p^{\varepsilon}(t, x)-\frac{1}{\varepsilon^{3}} \int V(x)\left|u^{\varepsilon}(t, x)\right|^{2}-\mathcal{E}(r) \\
& =m|v(t)|^{2}-v(t) \int p^{\varepsilon}(t, x)+m V(x(t))-\frac{1}{\varepsilon^{3}} \int V(x)\left|u^{\varepsilon}(t, x)\right|^{2}+\mathcal{O}\left(\varepsilon^{2}\right) .
\end{aligned}
$$

In turn $0 \leq \mathcal{E}\left(\Psi^{\varepsilon}(t)\right)-\mathcal{E}(r) \leq C \eta^{\varepsilon}(t)+\mathcal{O}\left(\varepsilon^{2}\right)$, where $\eta^{\varepsilon}$ is defined in [14, p.179] and satisfies $\eta^{\varepsilon}(0)=\mathcal{O}\left(\varepsilon^{2}\right)$. By Theorem 1.1 we know that there exist $C, A>0$ such that

$$
\mathcal{E}(\phi)-\mathcal{E}(r) \geq C \inf _{x \in \mathbb{R}^{3}, \theta \in[0,2 \pi)}\left\|\phi-e^{i \theta} r(\cdot-x)\right\|^{2}
$$

for any $\phi \in H^{1}\left(\mathbb{R}^{3}, \mathbb{C}\right)$ such that $\|\phi\|_{2}=\|r\|_{2}, \inf _{x \in \mathbb{R}^{3}, \theta \in[0,2 \pi)}\left\|\phi-e^{i \theta} r(\cdot-x)\right\| \leq\|r\|$ and $\mathcal{E}(\phi)-\mathcal{E}(r) \leq A$. Then, introducing

$$
T^{\varepsilon}=\sup \left\{t \in\left[0, T_{0}\right]: \eta^{\varepsilon}(s) \leq A, \inf _{x \in \mathbb{R}^{3}, \theta \in[0,2 \pi)}\left\|\Psi^{\varepsilon}(s, \cdot)-e^{i \theta} r(\cdot-x)\right\| \leq\|r\|, \text { for all } s \in[0, t]\right\}
$$

and observing that $\Psi^{\varepsilon}(0, x)=r(x)$, it follows that $T^{\varepsilon}>0$ for any $\varepsilon$ sufficiently small and there exist families of functions $\theta^{\varepsilon}:[0,2 \pi) \rightarrow \mathbb{R}$ and $z^{\varepsilon}: \mathbb{R}^{3} \rightarrow \mathbb{R}$ such that

$$
\left\|u^{\varepsilon}(t, x)-e^{\frac{i}{\varepsilon}\left(x \cdot v(t)+\theta^{\varepsilon}(t)\right)} r\left(\frac{x-z^{\varepsilon}(t)}{\varepsilon}\right)\right\|_{\mathcal{H}_{\varepsilon}}^{2} \leq C \eta^{\varepsilon}(t)+\mathcal{O}\left(\varepsilon^{2}\right), \quad \text { for all } t \in\left[0, T^{\varepsilon}\right) .
$$

From this stage on, taking into account the mass and momentum identities of Lemma 3.3, the conclusion $\eta^{\varepsilon}(t) \leq C \varepsilon^{2}$ for all $t \in\left[0, T^{\varepsilon}\right)$, and hence in turn for any $t \in\left[0, T_{0}\right]$, follows exactly as in [5, Lemma 3.4-3.6]. The conclusion of Lemma 3.2 is used in the proof of [5, Lemma 3.5] to have $\left\|p^{\varepsilon}(t)\right\|_{1} \leq C$ and choose in turn $T_{0}$ sufficiently small. Finally the assertion of Theorem 1.2 follows by mimicking the continuation argument exploited in [5, p.185].

\section{REFERENCES}

[1] A. Ambrosetti, A. Malchiodi, Concentration phenomena for nonlinear Schrödinger equations: recent results and new perspectives. Perspectives in nonlinear partial differential equations, 19-30, Contemp. Math., 446, Amer. Math. Soc., Providence, RI, 2007. 1

[2] V. Benci, M.G. Ghimenti, A.M. Micheletti, The nonlinear Schrödinger equation: soliton dynamics, J. Differential Equations 249 (2010), 3312-3341. 3

[3] V. Benci, M.G. Ghimenti, A.M. Micheletti, On the dynamics of solitons in the nonlinear Schröedinger equation, Arch. Ration. Mech. Anal. 205 (2012), 467-492. 3

[4] F.A. Berezin, M.A. Shubin, The Schrödinger equation. Translated from the 1983 Russian edition by Yu. Rajabov, D.A. Leites and N. A. Sakharova and revised by Shubin. With contributions by G.L. Litvinov and Leites. Mathematics and its Applications (Soviet Series), 66. Kluwer Academic Publishers Group, Dordrecht, 1991, 555pp. 8

[5] J.C. Bronski, R.L. Jerrard, Soliton dynamics in a potential, Math. Res. Lett. 7 (23) (2000), 329-342. 1, 2, 3, 12

[6] T. Cazenave, An introduction to nonlinear Schrödinger equations, Textos de Métodos Matemáticos 26, Universidade Federal do Rio de Janeiro 1996. 1, 2, 10, 11

[7] T. Cazenave, P.L. Lions, Orbital stability of standing waves for some nonlinear Schrdinger equations, Comm. Math. Phys. 85 (1982), 549-561. 1, 2

[8] S. Cingolani, S. Secchi, M. Squassina, Semi-classical limit for Schrödinger equations with magnetic field and Hartreetype nonlinearities, Proc. Roy. Soc. Edinburgh Sect. A 140 (2010), 973-1009. 1

[9] K. Datchev, I. Ventura, Solitary waves for the Hartree equation with a slowly varying potential, Pacific J. Math. 248 (2010), 63-90. 1

[10] J. Fröhlich, S. Gustafson, B.L.G. Jonsson, I.M. Sigal, Dynamics of solitary waves external potentials, Comm. Math. Phys. 250 (2004), 613-642. 1, 2, 11

[11] J. Fröhlich, Tai-Peng Tsai, Horng-Tzer Yau, On the point-particle (Newtonian) limit of the non-linear Hartree equation, Comm. Math. Phys. 225 (2002), 223-274. 2

[12] M. Grillakis, J. Shatah, W. Strauss, Stability theory of solitary waves in the presence of symmetry. I, J. Funct. Anal. $\mathbf{7 4}$ (1987), 160-197. 1 
[13] M. Grillakis, J. Shatah, W. Strauss, Stability theory of solitary waves in the presence of symmetry. II, J. Funct. Anal. 94 (1990), 308-348. 1

[14] S. KeraAni, Semiclassical limit for nonlinear Schrödinger equation with potential II, Asymptot. Anal. 47 (2006), 171-186. $1,2,3,12$

[15] E. Lenzmann, Uniqueness of ground states for pseudorelativistic Hartree equations, Anal. PDE 2 (2009), 1-27. 3, 4

[16] E.H. Lieb, Existence and uniqueness of the minimizing solution of Choquard's nonlinear equation, Stud. Appl. Math. 57 (1977), 93-105. 1, 3

[17] E.H. Lieb, M. Loss, Analysis. Second edition. Graduate Studies in Mathematics, 14. American Mathematical Society, Providence, RI, 2001, 346pp. 5, 8

[18] P.-L. Lions, The concentration-compactness principle in the calculus of variations. The locally compact case. I, Ann. Inst. H. Poincaré Anal. Non Linéaire 1 (1984), 109-145. 1, 2

[19] P.-L. Lions, The concentration-compactness principle in the calculus of variations. The locally compact case. II, Ann. Inst. H. Poincaré Anal. Non Linéaire 1 (1984), 223-283. 1, 2

[20] L. MA, L. Zhао, Classification of positive solitary solutions of the nonlinear Choquard equation, Arch. Ration. Mech. Anal. 195 (2010), 455-467. 3

[21] E. Montefusco, B. Pellacci, M. Squassina, Energy convexity estimates for non-degenerate ground states of nonlinear 1D Schrödinger systems, Commun. Pure Appl. Anal. 9 (2010), 867-884. 2

[22] V. Moroz, J. Van Schaftingen, Groundstates of nonlinear Choquard equations: existence, qualitative properties and decay asymptotics, preprint. 1

[23] S. Pekar, Untersuchung uber die Elektronentheorie der Kristalle, Akademie Verlag, Berlin, 1954. 1

[24] R. Penrose, Quantum computation, entanglement and state reduction, Phil. Trans. R. Soc. 356 (1998), 1-13. 1

[25] T. TAO, Why are solitons stable? Bull. Amer. Math. Soc. 46 (2009) 1-33. 2

[26] P. Tod, I. Moroz, An analytical approach to the Schrödinger-Newton equations, Nonlinearity 12 (1999), 201-216. 3

[27] J. Wei, M. Winter, Strongly interacting bumps for the Schrödinger-Newton equations, J. Math. Phys. 50 (2009), 22 pp 2

[28] M.I. Weinstein, Modulational stability of ground states of nonlinear Schrdinger equations, SIAM J. Math. Anal. 16 (1985) 472-491. 1, 2

[29] M.I. Weinstein, Lyapunov stability of ground states of nonlinear dispersive evolution equations, Comm. Pure Appl. Math. 39 (1986), 51-67. 1, 2

Dipartimento di Meccanica, Matematica e Management

POLITECNico Di BARI

Via Orabona 4, 70125 Bari, Italy

E-mail address: p.davenia@poliba.it

Dipartimento Di InFormatica

Università degLi Studi di Verona

Cá Vignal 2, Strada Le Grazie 15, 37134 Verona, Italy

E-mail address: marco.squassina@univr.it 